Siberian Mathematical Journal, Vol. 46, No. 3, pp. 2005

Original Russian Text Copyright (c) 2005 Goncharov S. S., Harizanov V. S., Knight J. F., Morozov A. S., Romina A. V.

\title{
ON AUTOMORPHIC TUPLES OF ELEMENTS IN COMPUTABLE MODELS
}

\section{S. S. Goncharov, V. S. Harizanov, J. F. Knight, A. S. Morozov, and A. V. Romina}

UDC 510.8

\begin{abstract}
A criterion is obtained for existence of two isomorphic but not hyperarithmetically isomorphic tuples in a hyperarithmetical model. This criterion is used to show that such a situation occurs in the models of well-known classes.
\end{abstract}

Keywords: model, computability, computable model, hyperarithmetical model, automorphism, recursive automorphism, admissible sets, recursive model, constructive model, Scott rank, quantifier rank, automorphic tuples

\section{Introduction}

We assume the reader to be familiar with the admissible sets (see [1]) and computable models (for instance, see [2] or [3]).

Let $\mathfrak{M}$ be a model whose basic set is a subset of $\omega$. We say that two tuples $\bar{a}, \bar{b} \in \mathfrak{M}<\omega$ are automorphic (hyperarithmetically automorphic) and denote this fact by $\bar{a} \cong \bar{b}\left(\bar{a} \cong_{h} \bar{b}\right)$, if there exists a (hyperarithmetical) automorphism $f$ of the model $\mathfrak{M}$ which takes elements $\bar{a}$ to elements of $\bar{b}$ correspondingly. Since it is always clear which model $\mathfrak{M}$ is meant, we will not mention models in our notations.

There exist a computable model $\mathfrak{M}$ and elements $a$ and $b$ of $\mathfrak{M}$ such that for some $f \in \operatorname{Aut}(\mathfrak{M})$ we have $f(a)=b$ but this property fails to be true for all hyperarithmetical automorphisms [4] (which follows also from [5]). In this paper, we will study these phenomena and related effects and show that this situation occurs quite often. Actually, we will prove a criterion for existence of such pairs. As its corollaries, we will obtain a series of examples of automorphic but not hyperarithmetically automorphic tuples in the models of well-known classes.

By $\mathbb{H} Y \mathbb{P}_{\omega}$ we denote the model $\mathbb{H} \mathbb{Y} \mathbb{P}_{\langle\omega,<\rangle}$ that is the least admissible set over $\langle\omega,<\rangle$ containing its basic set as an element.

We will need the following results.

Theorem (Barwise Compactness Theorem [1, Theorem III 5.6]). Let $L_{\mathbb{A}}$ be a countable admissible fragment of $L_{\infty, \omega}$ and let $T$ be a set of sentences of $L_{\mathbb{A}}$ which is a $\Sigma_{1}$-subset of $\mathbb{A}$. If each $T_{0} \subseteq T$ which is an element of $\mathbb{A}$ has a model then $T$ has a model.

Theorem 1.2. Assume that $\mathfrak{M} \in \mathbb{H} \mathbb{Y P}_{\omega}, \bar{a}, \bar{b} \in \mathfrak{M}^{n}$ and $\mathfrak{M} \models \varphi(\bar{a}) \Leftrightarrow \mathfrak{M} \models \varphi(\bar{b})$, for all $\varphi(\bar{x}) \in$ $\mathbb{H} \mathbb{Y} \mathbb{P}_{\omega}$. Then $\langle\mathfrak{M}, \bar{a}\rangle \cong\langle\mathfrak{M}, \bar{b}\rangle$.

Proof. It is immediate from Theorems VII 7.3, VII 5.2, and VII 5.3 of [1].

Theorem 1.3 [1, Theorems IV 3.1 and IV 3.7]. Let $\mathfrak{M}$ be a countable model and let $S$ be a relation on $\mathfrak{M}$. Then $S$ is a $\Pi_{1}^{1}$-relation on $\mathfrak{M}$ if and only if $S$ is a $\Sigma$-relation on $\mathbb{H} \mathbb{Y} \mathbb{P}_{\mathfrak{M}}$.

Note that if $\mathfrak{M}$ is a computable model then $\mathfrak{M} \in \mathbb{H} Y \mathbb{P}_{\omega}$. In this case we may think of the model $\mathfrak{M}$ as a structure whose basic set is an initial segment of the ordinal $\omega \in \mathbb{H} Y \mathbb{P}_{\omega}$.

The first four authors are supported by the Binational Grant NSF DMS-0075899; the first and fourth authors are partially supported by the Russian Foundation for Basic Research (Grant 02-01-00953) and the State Maintenance Program for the Leading Scientific Schools of the Russian Federation (Grant NSh-2112.03.1).

Novosibirsk; Washington; Notre Dame; Saarbrücken. Translated from Sibirskiu Matematicheskiu Zhurnal, Vol. 46, No. 3, pp. 523-532, May-June, 2005. Original article submitted November 10, 2004. 
Note that each $\Sigma$-subset of the ordinal $\omega \in \mathbb{H} \mathbb{Y} \mathbb{P}_{\omega}$ is a $\Pi_{1}^{1}$-subset of $\omega$. This follows from Theorem 1.3 and the existence of the bijection $f \in \mathbb{H} Y \mathbb{P}_{\omega}$ from $\omega$, which is considered as a model forming the "bottom" of the structure $\mathbb{H} \mathbb{Y} \mathbb{P}_{\omega}$, onto the set $\omega \in \mathbb{H} \mathbb{Y} \mathbb{P}_{\omega}$.

Recall the definition of the quantifier rank of an infinitary formula [1] (we assume that the implication $\Rightarrow$ is expressed via $\neg$ and $\wedge$, and by this it does not occur in our formulas):

$$
\operatorname{qr}(\varphi)= \begin{cases}0, & \text { if } \varphi \text { is quantifier-free; } \\ \operatorname{qr}(\psi), & \text { if } \varphi \text { has the form } \neg \psi ; \\ \operatorname{qr}(\psi)+1, & \text { if } \varphi \text { has the form } \exists v \psi \text { or } \forall v \psi ; \\ \sup \{\operatorname{qr}(\psi) \mid \psi \in \Phi\}, & \text { if } \varphi \text { has the form } \bigwedge \Phi \text { or } \bigvee \Phi\end{cases}
$$

Let $\alpha$ be an ordinal. Models $\mathfrak{M}$ and $\mathfrak{N}$ are called equivalent ( $\alpha$-equivalent) if they satisfy the same sentences (whose quantifier rank does not exceed $\alpha$ ). Denote this fact by $\mathfrak{M} \equiv \mathfrak{N}\left(\mathfrak{M} \equiv{ }^{\alpha} \mathfrak{N}\right)$. Two tuples $\bar{a}, \bar{b} \in \mathfrak{M}^{<\omega}$ are called equivalent ( $\alpha$-equivalent) if they satisfy the same sentences (of quantifier rank no more than $\alpha)$. This fact is denoted by $\bar{a} \equiv \bar{b}\left(\bar{a} \equiv^{\alpha} \bar{b}\right)$. We say that $\bar{a} \in \mathfrak{M}^{<\omega}$ has quantifier rank $\alpha$ in $\mathfrak{M}$ if the implication $\left(\bar{a} \equiv^{\alpha} \bar{b} \Rightarrow \bar{a} \equiv \bar{b}\right)$ holds for all $\bar{b} \in \mathfrak{M}^{<\omega}$ and $\alpha$ is the smallest ordinal with this property. It is denoted by $\operatorname{qr}(\bar{a})$; the Barwise rank $\operatorname{br}(\mathfrak{M})$ of a model $\mathfrak{M}$ is the smallest ordinal $\alpha$ such that $\left(\bar{a} \equiv^{\alpha} \bar{b} \Rightarrow \bar{a} \equiv^{\alpha+1} \bar{b}\right)$ for all $\bar{a}, \bar{b} \in \mathfrak{M}^{<\omega}$. This is exactly what is called the Scott rank in [1]. It is well known that the Barwise rank of $\mathfrak{M} \in \mathbb{H} \mathbb{Y} \mathbb{P}_{\omega}$ does not exceed $\omega_{1}^{\mathrm{CK}}[1]$.

We will also need the existence of the series of formulas $\sigma_{\mathfrak{M}, \bar{a}}^{\alpha}(\bar{x}) \in \mathbb{H} \mathbb{Y} \mathbb{P}_{\omega}$; where $\mathfrak{M}$ is a model, $\alpha$ is an ordinal, and $\bar{a} \in \mathfrak{M}^{<\omega}$ with the following properties:

(1) formulas $\sigma_{\mathfrak{M}, \bar{a}}^{\alpha}$ are defined by means of a $\Sigma$-function on $\mathbb{H} \mathbb{Y} \mathbb{P}_{\omega}$ from $\alpha<\omega_{1}^{\mathrm{CK}}, \mathfrak{M} \in \mathbb{H} \mathbb{Y} \mathbb{P}_{\omega}$, and $\bar{a} \in \mathfrak{M}^{<\omega}$

(2) for all $\bar{a}, \bar{b} \in \mathfrak{M}^{<\omega}$, the statement $\mathfrak{M}=\sigma_{\mathfrak{M}, \bar{a}}^{\alpha}(\bar{b})$ is true if and only if $\bar{a} \equiv^{\alpha} \bar{b}$;

(3) $\operatorname{qr} \sigma_{\mathfrak{M}_{j} \bar{a}}^{\alpha}=\alpha$.

We will omit the index $\mathfrak{M}$ and simply write $\sigma_{\bar{a}}^{\alpha}$ because it is always which model $\mathfrak{M}$ is meant.

About the general results on using ordinals in analysis of structures also see [6].

\section{The General Result}

Theorem 2.1. Let $\mathfrak{M}$ be a hyperarithmetical model. Then the following conditions are equivalent:

(1) there exist tuples $\bar{a}, \bar{b} \in \mathfrak{M}^{<\omega}$ such that $\langle\mathfrak{M}, \bar{a}\rangle \cong\langle\mathfrak{M}, \bar{b}\rangle$, but $\langle\mathfrak{M}, \bar{a}\rangle \varlimsup_{h}\langle\mathfrak{M}, \bar{b}\rangle$;

(2) there exists a tuple $\bar{a} \in \mathfrak{M}^{<\omega}$ admitting an infinite family $\left(\bar{a}_{i}\right)_{i<\omega}$ of tuples in $\mathfrak{M}<\omega$ with the following properties:

(a) $\langle\mathfrak{M}, \bar{a}\rangle \cong\left\langle\mathfrak{M}, \bar{a}_{i}\right\rangle$ for all $i<\omega$,

(b) $\left\langle\mathfrak{M}, \bar{a}_{i}\right\rangle \nsupseteq_{h}\left\langle\mathfrak{M}, \bar{a}_{j}\right\rangle$ for all $i<j<\omega$;

(3) the Barwise rank of $\mathfrak{M}$ equals $\omega_{1}^{\mathrm{CK}}$;

(4) $I_{\mathfrak{M}} \notin \Pi_{1}^{1}$, where $I_{\mathfrak{M}}=\left\{\langle\bar{a}, \bar{b}\rangle \in \mathfrak{M}^{<\omega} \times \mathfrak{M}^{<\omega} \mid \bar{a} \cong \bar{b}\right\}$.

Proof. The implication $2 \Rightarrow 1$ is obvious.

$1 \Rightarrow 4$. To obtain a contradiction, assume that $I_{\mathfrak{M}} \in \Pi_{1}^{1}$. Then $I_{\mathfrak{M}}$ is in $\Delta_{1}^{1}$ because $I_{\mathfrak{M}}$ is always in $\Sigma_{1}^{1}$. Using the usual back-and-forth argument with $I_{\mathfrak{M}}$ as an oracle, we can easily construct a hyperarithmetical automorphism taking $\bar{a}$ to $\bar{b}$, which is a contradiction. We start with a finite automorphism $f_{0}=f$ that takes $\bar{a}$ to $\bar{b}$. At each step, we use the oracle $I_{\mathfrak{M}}$ to extend the current values of tuples by adding finite families of elements to its domain and range so that the resulting pair of these new tuples will be in $I_{\mathfrak{M}}$. At each step, we extend the domain of $f$ by the least element which is still not in it. Then we do the same for the range of $f$.

$4 \Rightarrow 3$. Suppose that $I_{\mathfrak{M}} \notin \Pi_{1}^{1}$, but the Barwise rank of $\mathfrak{M}$ is less than $\omega_{1}^{\mathrm{CK}}$. Denote it by $\alpha$. In this case, from the definition of Barwise rank we find that the following conditions are equivalent:

(1) $\bar{a} \cong \bar{b}$

(2) $\mathfrak{M} \models \sigma_{\mathfrak{M}, \bar{a}}^{\alpha}[\bar{b}]$.

It implies that $I_{\mathfrak{M}}$ belongs to $\Delta_{1}^{1}$, which contradicts the assumption we made before. 
$3 \Rightarrow 2$. Assume for a contradiction that for each tuple $\bar{a} \in \mathfrak{M}^{<\omega}$ the number of types of hyperarithmetical isomorphism within its orbit is finite; namely,

$$
\forall \bar{a}_{1} \in \mathfrak{M}^{<\omega} \exists t \in \omega \forall \bar{a}_{2} \ldots \bar{a}_{t}\left(\bigwedge_{i=2}^{t} \bar{a}_{1} \cong \bar{a}_{i} \Rightarrow \bigvee_{1 \leq i<j \leq t} \bar{a}_{i} \cong_{h} \bar{a}_{j}\right) .
$$

This could be rewritten as:

$$
\forall \bar{a}_{1} \in \mathfrak{M}^{<\omega} \exists t \in \omega \forall \bar{a}_{2} \ldots \bar{a}_{t} \exists \beta\left(\bigvee_{i=2}^{t} \bar{a}_{1} \not{ }^{\beta} \bar{a}_{i} \vee \bigvee_{1 \leq i<j \leq t} \bar{a}_{i} \cong_{h} \bar{a}_{j}\right) .
$$

(Here we use natural abbreviations like

$$
\begin{gathered}
\bigvee_{i=2}^{t} \bar{a}_{1} \equiv^{\beta} \bar{a}_{i} \quad \text { for } \exists i \leq t\left(i \geq 2 \& \bar{a}_{1} \not^{\beta} \bar{a}_{i}\right), \\
\left.\forall \bar{a}_{2} \ldots \bar{a}_{t}(\ldots) \text { for } \forall m\left(\left(m \text { codes }\left\langle\bar{a}_{2}, \ldots, \bar{a}_{t}\right\rangle\right) \Rightarrow(\ldots)\right) \text { etc. }\right)
\end{gathered}
$$

After the double application of $\Sigma$-collection, we obtain the existence of a $\alpha<\omega_{1}^{\mathrm{CK}}$ such that

$$
\forall \bar{a}_{1} \in \mathfrak{M}^{<\omega} \exists t \in \omega \forall \bar{a}_{2} \ldots \bar{a}_{t}\left(\bigwedge_{i=2}^{t} \bar{a}_{1} \equiv^{\alpha} \bar{a}_{i} \Rightarrow \bigvee_{1 \leq i<j \leq t} \bar{a}_{i} \cong_{h} \bar{a}_{j}\right)
$$

Prove that the Barwise rank of $\mathfrak{M}$ does not exceed $\alpha+\omega$, which will contradict the initial assumption. In fact, we will prove that if some tuples $\bar{a}, \bar{b} \in \mathfrak{M}^{<\omega}$ satisfy $\bar{a} \equiv^{\alpha+\omega} \bar{b}$ then $\bar{a} \cong \bar{b}$. To prove this, it suffices to check that if $\bar{a} \equiv^{\alpha+\omega} \bar{b}$ then for each $c$ there is a $d$ such that $\bar{a} c \equiv^{\alpha+\omega} \bar{b} d$, and the statement will follow from the back-and-forth argument.

Assume that $\bar{a} \equiv^{\alpha+\omega} \bar{b}$. Take an arbitrary element $c$. Then the statement $\sigma_{\bar{a} c}^{\alpha}(\bar{x}, y)$ about $\bar{a} c$ is true in $\mathfrak{M}$. Henceforth, $\mathfrak{M} \models \exists y \sigma_{\bar{a} c}^{\alpha}(\bar{a}, y)$. From $\bar{a} \equiv^{\alpha+\omega} \bar{b}$, it follows that $\mathfrak{M} \models \exists y \sigma_{\bar{a} c}^{\alpha}(\bar{b}, y)$. From here we obtain that the set

$$
D=\left\{p \mid \mathfrak{M}=\sigma_{\bar{a} c}^{\alpha}(\bar{b}, p)\right\}
$$

is nonempty. It is obvious that each element $p \in D$ satisfies $\bar{a} c \equiv^{\alpha} \bar{b} p$.

Note that the subsets of $D$ definable by the formulas whose quantifier rank is less than $\alpha+\omega$ with parameters $\bar{b}$ form a finite Boolean algebra. Otherwise there would be infinitely many pairwise $\alpha$-equivalent nonautomorphic tuples of kind $\bar{b} p, p \in D$, which contradicts $(1)$. Let the formulas $\varphi_{1}(\bar{b}, y), \ldots, \varphi_{k}(\bar{b}, y)$ of quantifier rank less than $\alpha+\omega$ define the atoms of the above-mentioned Boolean algebra. If there is no $i \in\{1, \ldots, k\}$ such that $c$ satisfies $\varphi_{i}(\bar{a}, y)$ then

$$
\mathfrak{M} \models \exists y\left(\bigwedge_{i=1, \ldots, k} \neg \varphi_{i}(\bar{a}, y) \& \sigma_{\bar{a} c}^{\alpha}(\bar{a}, y)\right) ;
$$

but this statement becomes false if we replace $\bar{a}$ with $\bar{b}$; this contradicts $\bar{a} \equiv^{\alpha+\omega} \bar{b}$. Choose $i$ so that to satisfy $\mathfrak{M} \models \varphi_{i}(\bar{a}, c)$. Take an arbitrary $d$ in $D$ that satisfies the formula $\varphi_{i}(\bar{b}, x)$ in $\mathfrak{M}$ and check the equivalence $\bar{a} c \equiv^{\alpha+\omega} \bar{b} d$. Suppose it false and let $\psi(\bar{x}, y)$ be a formula of quantifier rank less than $\alpha+\omega$ which distinguishes these tuples; i.e., $\mathfrak{M} \models \psi(\bar{a}, c)$ but $\mathfrak{M} \models \neg \psi(\bar{b}, d)$. Inasmuch as the formulas $\varphi_{i}(\bar{b}, y)$, $1 \leq i \leq k$, define atoms, we have

$$
\mathfrak{M} \models \forall y\left(\sigma_{\bar{a} c}^{\alpha}(\bar{b}, y) \& \varphi_{i}(\bar{b}, y) \Rightarrow \neg \psi(\bar{b}, y)\right)
$$

but

$$
\mathfrak{M} \models \exists y\left(\sigma_{\bar{a} c}^{\alpha}(\bar{a}, y) \& \varphi_{i}(\bar{a}, y) \& \psi(\bar{a}, y)\right) ;
$$

this contradicts the equivalence $\bar{a} \equiv^{\alpha+\omega} \bar{b}$. 
Theorem 2.2. Let $\mathfrak{M}$ be a hyperarithmetical model and $\bar{a}=a_{1}, \ldots, a_{k} \in \mathfrak{M}^{<\omega}$. Then $\operatorname{qr}(\bar{a})=\omega_{1}^{\mathrm{CK}}$ is true if and only if $I_{\bar{a}} \notin \Pi_{1}^{1}$, where $I_{\bar{a}}={ }_{\operatorname{def}}\left\{\bar{b} \in \mathfrak{M}^{<\omega} \mid \bar{a} \cong \bar{b}\right\}$.

Proof. $(\Rightarrow)$ Assume for a contradiction that $I_{\bar{a}} \in \Pi_{1}^{1}$.

Here we use the Barwise Compactness Theorem. Let $L$ be the language of $\mathfrak{M}$. Extend it to the language $L^{\prime}$ by adding the new constant 0 which is interpreted as zero, the constants $\bar{c}=c_{0}, \ldots, c_{k}$ and a new unary functional symbol $s$ which is understood to be increasing by one. We will construct a $\Sigma$-family $T$ of sentences of the fragment $L^{*}=L_{\omega_{1} \omega}^{\prime} \cap \mathbb{H} \mathbb{Y} \mathbb{P}_{\omega}$ which describes some enrichment of $\mathfrak{M}$ and states that $\mathfrak{M}$ satisfies $\bar{c} \cong \bar{a}$ and, simultaneously, $\bar{c} \notin I_{\bar{a}}$. Then we will show that each part of $T$ belonging to $\mathbb{H} Y \mathbb{P}_{\omega}$ has a model. It is clear that the family $T$ cannot be compatible. Nevertheless, by the Barwise Compactness Theorem $T$ has a model and is thus compatible, which is a contradiction.

Let $T=S_{0} \cup S_{1} \cup S_{2} \cup S_{3}$, where

- $S_{0}$ is the set $\left\{\forall x \bigvee_{m \in \omega}\left(x=s^{m}(0)\right)\right\}$ (each element of $\mathfrak{M}$ is a natural number);

- $S_{1}$ is the diagram of $\mathfrak{M}$ in the language $L \cup\{s, 0\}$; i.e., the family

$$
\left\{\varphi\left(s^{m_{1}}(0), \ldots, s^{m_{l}}(0)\right) \mid l, m_{1}, \ldots, m_{l} \in \omega, \varphi\left(x_{1}, \ldots, x_{l}\right)\right.
$$

is a quantifier-free formula of $L$ such that $\left.\mathfrak{M} \models \varphi\left(m_{1}, \ldots, m_{l}\right)\right\}$;

- $S_{2}=\left\{\neg \bigvee_{i=1, \ldots, k}\left(c_{i}=s^{b_{i}}(0)\right) \mid \bar{b}=b_{1}, \ldots, b_{k} \in I_{\bar{a}}\right\} \quad\left(\bar{c}\right.$ does not coincide with any tuple $\left.\bar{b} \in I_{\bar{a}}\right)$; this is a $\Sigma$-family on $\mathbb{H} \mathbb{Y} \mathbb{P}_{\omega}$ since we have assumed that $I_{\bar{a}} \in \Pi_{1}^{1}$;

- $S_{3}=\left\{\varphi(\bar{c}) \mid \varphi(\bar{x}) \in L^{*} \& \mathfrak{M}=\varphi(\bar{a})\right\}\left(\bar{c}\right.$ and $\bar{a}$ satisfy the same formulas of $L^{*}$, henceforth they are isomorphic in $\mathfrak{M}$ by Theorem 1.2).

Check that each part of $T$ which belongs to $\mathbb{H} \mathbb{P}_{\omega}$ has a model. Indeed, each $S \subseteq S_{3}$ such that $S \in \mathbb{H} \mathbb{Y} \mathbb{P}_{\omega}$ satisfies the condition

$$
A_{S}=\left\{\bar{d} \mid \mathfrak{M} \models \bigwedge_{\varphi(\bar{c}) \in S} \varphi(\bar{d})\right\} \backslash I_{\bar{a}} \neq \varnothing .
$$

Otherwise the set $I_{\bar{a}}$ could be defined by a formula of the fragment $L^{*}$, which would imply $\operatorname{qr}(\bar{a})<$ $\omega_{1}^{\text {CK }}$. Every tuple of elements of $A_{S}$ could be taken as an interpretation of $\bar{c}$. The model $\mathfrak{M}$ with such an interpretation for $\bar{c}$ along with the obvious interpretations for $s$ and 0 is a model of the family $S_{0} \cup S_{1} \cup S_{2} \cup S$.

By the Barwise Compactness Theorem, $T$ has a model. Since this model satisfies $S_{0}$ and $S_{1}$, its restriction to the language $L$ is isomorphic to $\mathfrak{M}$. (If we identify $s^{p}(0)$ with $p$ for all $p<\omega$, it will be actually the same as $\mathfrak{M}$.) The families $S_{2}$ and $S_{3}$ assert that $\bar{c} \notin I_{\bar{a}}$ but $\bar{c} \cong \bar{a}$. We have arrived at a contradiction.

$(\Leftarrow)$ If $\operatorname{qr}(\bar{a})<\omega_{1}^{\mathrm{CK}}$ then $\forall \bar{b} \in \mathfrak{M}^{k}\left(\bar{a} \cong \bar{b} \Leftrightarrow \mathfrak{M}=\sigma_{\mathfrak{M}, \bar{a}}^{q r(\bar{a})}[\bar{b}]\right)$. It follows that $I_{\bar{a}}$ is a $\Delta$-subset of $\mathbb{H} \mathbb{Y} \mathbb{P}_{\omega} ;$ i.e., $I_{\bar{a}} \in \Pi_{1}^{1}$, which leads to a contradiction.

REMARK. It can happen that two automorphic tuples of quantifier rank less than $\omega_{1}^{\mathrm{CK}}$ cannot be identified by means of a hyperarithmetical automorphism. Here is an easy example. Take two isomorphic but not hyperarithmetically isomorphic models $\mathfrak{M}_{0}$ and $\mathfrak{M}$ whose basic sets are $\omega$ and whose language $L$ contains only predicate symbols. For instance, we can take some models of kind $\langle\mathfrak{M}, \bar{a}\rangle$ and $\langle\mathfrak{M}, \bar{b}\rangle$, where $\bar{a}$ and $\bar{b}$ are automorphic but not hyperarithmetically automorphic in $\mathfrak{M}$ and transform them into models of predicate signatures. The existence of such models is shown above. Consider a new model $\mathfrak{M}^{*}$ that is a direct sum of the models $\mathfrak{M}_{0}$ and $\mathfrak{M}_{1}$ with two new elements $a_{0}$ and $a_{1}$ and a new predicate $P$ such that

$$
P(x, y) \Leftrightarrow\left(\left(x=a_{0}\right) \&\left(y \in \mathfrak{M}_{0}\right)\right) \vee\left(\left(x=a_{1}\right) \&\left(y \in \mathfrak{M}_{1}\right)\right) .
$$

Define a new computable model $\mathfrak{M}$ whose structure is transferred to $\omega$ by means of a mapping

$$
f(x)= \begin{cases}a_{0}, & \text { if } x=0, \\ a_{1}, & \text { if } x=1, \\ t \in \mathfrak{M}_{0}, & \text { if } x=2 t+2, \\ t \in \mathfrak{M}_{1}, & \text { if } x=2 t+3\end{cases}
$$


It is easy to ascertain that $f^{-1}\left(a_{0}\right)$ and $f^{-1}\left(a_{1}\right)$ are automorphic but not hyperarithmetically automorphic in $\mathfrak{M}$ and the set $\left\{f^{-1}\left(a_{0}\right), f^{-1}\left(a_{1}\right)\right\}$ is definable by a formula $\exists y P(x, y)$, which implies that the quantifier rank of $f^{-1}\left(a_{0}\right)$ and $f^{-1}\left(a_{1}\right)$ is finite.

Theorem 2.3. Let $\varphi$ be a sentence in $L_{\mathbb{H} Y \mathbb{P}_{\omega}}$. Let $m<\omega$ and let $A$ be a hyperarithmetical subset of $\omega$ such that for each constructive ordinal $\alpha$ there exist an $A$-computable model $\mathfrak{A} \models \varphi$ and tuples $\bar{a}, \bar{b} \in \mathfrak{A}^{m}$ such that $\langle\mathfrak{A}, \bar{a}\rangle \cong\langle\mathfrak{A}, \bar{b}\rangle$ but there is no automorphism of $\mathfrak{A}$ which is computable in $\mathbf{0}^{(\alpha)}$ and taking $\bar{a}$ to $\bar{b}$. Then there exists an $A$-computable model $\mathfrak{B} \vDash \varphi$ and $\bar{a}, \bar{b} \in \mathfrak{B}^{m}$ such that $\langle\mathfrak{B}, \bar{a}\rangle \cong\langle\mathfrak{B}, \bar{b}\rangle$, but $\langle\mathfrak{B}, \bar{a}\rangle \oiint_{h}\langle\mathfrak{B}, \bar{b}\rangle$.

Proof. We give only a sketch. The result will follow from the Barwise Compactness Theorem. To apply this theorem, we define a $\Sigma$-family of sentences in $L_{\mathbb{H} Y \mathbb{P}_{\omega}}$ which states that:

(1) $n<\omega$ codes some $A$-computable model for $\varphi$;

(2) for all hyperarithmetical permutations $f$ on $\omega$ it is not true that $f$ is an automorphism of this model taking $\bar{a}$ to $\bar{b}$.

By the Barwise Compactness Theorem, there exists a natural number $n<\omega$ coding an $A$-computable model of $\varphi$ with the required property.

\section{Applications to Concrete Classes of Models}

LINEAR ORDERINGS. It is well known that there exists a computable linear ordering of type $\omega_{1}^{\mathrm{CK}}+$ $\left(\omega_{1}^{\mathrm{CK}} \times \eta\right)$, where $\eta$ is an order type of the rationals such that every two distinct automorphic elements from the second summand are not hyperarithmetically automorphic (see $[2,5,7]$ ). From here we can conclude that the Barwise rank of this ordering equals $\omega_{1}^{\mathrm{CK}}$.

$p$-Groups. Apply Theorem 2.1 to prove the existence of a computable $p$-group and two its automorphic but not hyperarithmetically automorphic tuples. To do so, we need to construct a computable $p$-group of Barwise rank $\omega_{1}^{\mathrm{CK}}$. But in this case we will not know the length of these tuples. We will give a direct proof of the existence of such a group and tuples of length 1. From here, it will follow the existence of a $p$-group of Barwise rank $\omega_{1}^{\mathrm{CK}}$.

Theorem 3.1. For each prime $p$, there exist a computable $p$-group and its elements $a$ and $b$ which are automorphic but not hyperarithmetically automorphic.

Proof. Given a constructive ordinal $\alpha$, we construct a computable $p$-group and its elements $a$ and $b$ which are automorphic but the Turing degree of each automorphism that takes $a$ to $b$ is greater or equal to $\mathbf{0}^{(\alpha)}$. This theorem will follow from Theorem 2.3. Recall some definitions first. Considering the presentations of groups by trees, see, for instance, [8].

A tree is an arbitrary subset $T \subseteq \omega^{<\omega}$ containing the empty sequence $\varnothing$ which is closed under initial segments; i.e., if $u \in T$ and $v$ is an initial segment of $u$ then $v \in T$. We assume that our trees are "growing down," for example, the sequence $\langle 1,2,3\rangle$ is situated below $\langle 1,2\rangle$. Each element of $s \in \omega^{<\omega}$ but $\varnothing$ has its predecessor that is obtained by removing the last symbol of $s$; for instance $\langle 0\rangle$ is the predecessor of $\langle 0,1\rangle$. The sequence $u=\left(u_{i}\right)_{i<\omega}$ of elements of a tree $T$ is called an infinite branch of $T$ if $u_{0}=\varnothing$ and its every element $u_{i}$ is the predecessor of $u_{i+1}$. We denote $u \uparrow i=u_{i}$. The derivative of a tree $T$ is the set

$$
T^{\prime}=\{s \in T \mid s \text { is the predecessor of some element of } T\} \cup\{\varnothing\} .
$$

For an arbitrary ordinal $\alpha$, define by induction the $\alpha$ th derivative $T^{(\alpha)}$ of $T$ as follows:

$$
\begin{aligned}
T^{(0)} & =T ; \\
T^{(\alpha+1)} & =\left(T^{(\alpha)}\right)^{\prime} ; \\
T^{(\gamma)} & =\bigcap_{\beta<\gamma} T^{(\beta)}, \text { if } \gamma \text { is a limit ordinal. }
\end{aligned}
$$


The level of an element $a \in T$ is the number of elements in the sequence $a$. Each well-founded element $a \in T$ has ordinal rank $\operatorname{rk}(a)$ which is defined as follows:

$$
\operatorname{rk}(a)=\sup \{\operatorname{rk}(b)+1 \mid a \text { is the predecessor of } b\} .
$$

It is easy to see that $\operatorname{rk}(a)=\min \left\{\alpha \mid a \in T^{(\alpha)} \backslash T^{(\alpha+1)}\right\}$.

Fix a prime $p$. With each tree $T$, we can associate an Abelian $p$-group $G[T]$ as follows: this group is defined by the following set of generators and defining relations:

$$
\{p a=b \mid a, b \in T \& b \text { is the predecessor of } a\} .
$$

The elements of the group $G[T]$ are denoted by the formal expressions of kind $\sum_{i=1}^{k} c_{i} a_{i}$, where $a_{i} \in T, c_{i} \in \mathbb{Z}$. We now describe canonical presentations of elements in $G[T]$. Consider the following three types of reductions.

I. $m a \rightarrow r a+q b$, where $b$ is the predecessor of $a$ and $m \neq 0, m=p q+r, q, r \in \mathbb{Z}, 0 \leq r<p$.

II. $\varnothing+a \rightarrow a$.

III. $0 a \rightarrow \varnothing$.

An application of each of these reductions to an expression of kind $\sum_{i=1}^{k} c_{i} a_{i}$ consists of the replacement of some of its subexpression which is the left-hand part of this reduction by the right-hand part of the reduction and further collecting summands so that to obtain the expression of the same type; while doing this, we do not care about the order of this summands. We can check that these reductions have the following properties:

(1) the result of application of a reduction denotes the same element as the initial expression;

(2) for each expression $S$, there is no infinite sequence of expressions $S=S_{0}, S_{1}, S_{2}, \ldots$ in which each element $S_{i+1}$ is obtained from $S_{i}$ by a reduction of type I, II, or III and $S_{i} \neq S_{i+1}, i=0,1,2, \ldots$;

(3) if a formal expression $S$ can be reduced by finite series of reductions to formal expressions $S^{\prime}$ and $S^{\prime \prime}$ then $S^{\prime}$ and $S^{\prime \prime}$ could be reduced by a finite series of reductions to the same formal expression $S^{\prime \prime \prime}$.

It follows that each sequence of reductions will stop at the situation when further reductions are impossible, and the result does not depend on the order of applications of reductions. The expressions that cannot be reduced are called canonical forms. We can consider the group $G[T]$ as the set of canonical forms on which the addition + is naturally defined as taking a formal sum and computing the canonical form of the so-obtained expression.

For each $\alpha$, define the subgroup $p^{\alpha} G \leq G$ as usual:

$$
\begin{aligned}
p^{0} G & =G ; \\
p^{\alpha+1} G & =\left\{x \in p^{\alpha} G \mid \exists y \in p^{\alpha} G(x=p y)\right\} ; \\
p^{\gamma} & =\bigcap_{\beta<\gamma} p^{\beta} G, \text { if } \gamma \text { is a limit ordinal. }
\end{aligned}
$$

Lemma 3.2. Assume that $T$ is a tree. Then the group $p^{\alpha} G[T]$ coincides with a subgroup of $G[T]$ generated by all elements of $T^{(\alpha)}$.

Proof. Induction on $\alpha$.

Fix a constructive ordinal $\alpha$ and consider a computable tree $T_{0}$ possessing a unique infinite branch $u$ whose Turing degree equals $\mathbf{0}^{(\alpha)}$. Such trees exist (see [9]). Define a tree $T_{1}$ as $\{\varnothing,\langle 0\rangle,\langle 0,0\rangle,\langle 0,0,0,\rangle \ldots\}$. This tree consists of a sole infinite branch. Define the computable tree $T$ as

$$
T=\{\varnothing\} \cup\left\{1 s \mid s \in T_{0}\right\} \cup\left\{2 s \mid s \in T_{1}\right\} .
$$

Note that this tree has exactly two infinite branches:

$$
s_{0}^{*}=\{\varnothing\} \cup\left\{1(u\lceil i) \mid i \in \omega\}, \quad s_{1}^{*}=\{\varnothing\} \cup\left\{2 s \mid s \in T_{1}\right\} .\right.
$$


Show that the elements $a=\langle 1\rangle$ and $b=\langle 2\rangle$ are automorphic in $G[T]$. Note that there exists an ordinal $\beta$ such that $T^{(\beta+1)}=T^{(\beta)}$. In addition, $T^{(\beta)}=s_{0}^{*} \cup s_{1}^{*}$ and $G\left[T^{(\beta)}\right]=G\left[s_{0}^{*}\right] \oplus G\left[s_{1}^{*}\right]$. Moreover, $G\left[T^{(\beta)}\right]$ is a divisible subgroup in $G[T]$. Since each divisible subgroup is a direct summand in every group (see [10, Theorem 21.2]), $G[T]$ has decomposition

$$
G[T]=G\left[s_{0}^{*}\right] \oplus G\left[s_{1}^{*}\right] \oplus H
$$

for some subgroup $H \leq G[T]$.

It is easy to see that $a$ and $b$ are automorphic in the sum $G\left[s_{0}^{*}\right] \oplus G\left[s_{1}^{*}\right]$. In accordance with (2), they are automorphic in $G[T]$ as well.

Every automorphism $f$ of $G[T]$ taking $a$ to $b$ has Turing degree greater or equal than $\mathbf{0}^{(\alpha)}$. Recall that the infinite branch $s_{1}^{*}$ consists of elements $\varnothing,\langle 2\rangle,\langle 2,0\rangle,\langle 2,0,0\rangle,\langle 2,0,0,0\rangle, \ldots$ Note that, inasmuch as $p \cdot\langle 2,0\rangle=\langle 2\rangle$, the canonical form $f^{-1}(\langle 2,0\rangle)$ contains an element of level 2 in $s_{0}^{*}$. By the same reasons, we see that the canonical form $f^{-1}(\langle 2,0,0\rangle)$ contains an element of $s_{0}^{*}$ of level 3, etc. Thus, the following algorithm computes $s_{0}^{*}$ with respect to $f$ : to compute $s_{0}^{*} \uparrow m$ just take an arbitrary element of a canonical presentation for $f^{-1}\left(\left\langle 2,0^{m}\right\rangle\right)$ which is situated below $s_{0}^{*}\lceil(m-1)$.

Now it remains to apply Theorem 2.3 .

Corollary 1. There exists a p-group of Barwise rank $\omega_{1}^{\mathrm{CK}}$.

Proof. See Theorem 2.1.

Boolean Algebras. Here we will prove the existence of Boolean algebras of Barwise rank $\omega_{1}^{\mathrm{CK}}$. Then we prove that the number of elements in hyperarithmetical nonautomorphic tuples can be equal to 1.

In [11] P. E. Alaev has proven the result implying that if $\mathfrak{B}$ is an $\alpha$-atomic Boolean algebra then its Barwise rank is greater or equal than $\alpha$. Although this estimation is rather rough, it suffices for our purposes. On the other hand, S. S. Goncharov in [12] has proven that for each constructive ordinal $\alpha<\omega_{1}^{\mathrm{CK}}$ there exists an $\alpha$-atomic computable Boolean algebra.

Using these results, we can construct a $\Sigma$-set $T$ of $L_{\mathbb{H Y Y}}$-formulas which state that a natural number $m$ is a code of a computable Boolean algebra whose Barwise rank is at least $\omega_{1}^{\mathrm{CK}}$.

Note first that there exists a $\Sigma$-function $\alpha \mapsto \Phi_{\alpha}(x)$ over $\mathbb{H} \mathbb{Y} \mathbb{P}_{\omega}$ which given an ordinal $\alpha<\omega_{1}^{\mathrm{CK}}$ outputs a formula saying that $x$ belongs to the $\alpha$ th Fréchet ideal. This function could be defined by induction as follows. Let

$$
\Phi_{0}(x)=\bigvee_{n \in \omega}\left(\bigwedge_{i=1, \ldots, n} \operatorname{atom}\left(x_{i}\right) \& \bigwedge_{1 \leq i<j \leq n} \neg\left(x_{i}=x_{j}\right) \& x=x_{1} \cup \cdots \cup x_{n}\right),
$$

where $\operatorname{atom}(x)$ is an abbreviation for $\forall y[(x \cap y=0) \vee(x \cap y=x)]$. The formula $\Phi_{\alpha+1}(x)$ is obtained from $\Phi_{\alpha}(x)$ by replacement of all occurrences of $A=B$ with an equality modulo $\alpha$ th Fréchet ideal $\mathscr{F}_{\alpha}$; i.e., by replacement with the formula $\Phi_{\alpha}((A \cap \bar{B}) \cup(B \cap \bar{A}))$. On limit steps $\beta$ we define $\Phi_{\beta}(x)$ as a conjunction of all formulas $\Phi_{\gamma}(x), \gamma<\beta$. One can verify by induction that the formula $\Phi_{\alpha}(x)$ defines in each Boolean algebra the $\alpha$ th Fréchet ideal.

Consider the language $L=\left\langle 0, s^{1},+^{2}, .^{2}, \cap^{2}, \cup^{2},{ }^{-1}\right\rangle$ and the standard model of arithmetics as its model in which the operations $0, s^{1},+^{2}, .^{2}$ have their usual interpretations.

Now we can describe the set $T$ of sentences. The sentences of the first part of $T$ state that elements of $s^{k}(0)$ form a standard model of arithmetics with usual operations,$+ \cdot$ and that $\cap^{2}, \cup^{2},-1$ are computable operations on $\omega$, and $\omega$ together with these operations forms a Boolean algebra.

The second part of $T$ is the family of sentences saying that for each $\alpha<\omega_{1}^{\mathrm{CK}}$ the set $\mathscr{F}_{\alpha+1} \backslash \mathscr{F}_{\alpha}$ is nonempty.

By the above, each part of $T$ which is an element of $\mathbb{H} \mathbb{Y} \mathbb{P}_{\omega}$ has a model. Henceforth, the whole set $T$ has a model. The set $T$ states that this model is computable. Its Barwise rank is exactly $\omega_{1}^{\mathrm{CK}}$, inasmuch as above it cannot be less than $\omega_{1}^{\mathrm{CK}}$ and, on the other hand, the Barwise rank of a computable model does not exceed $\omega_{1}^{\mathrm{CK}}$. 
Denote this Boolean algebra by $\mathfrak{B}$. By Theorem 2.1, there exist tuples of elements which are automorphic but not hyperarithmetically automorphic. It is clear that they should have nonzero length. Now we can reduce the number of elements in these tuples to 1 . We will use the following notation: $a^{0}=a$, $a^{1}=\bar{a}$. Since each hyperarithmetical isomorphism between two tuples $a_{1}, \ldots, a_{m}$ and $b_{1}, \ldots, b_{m}$ corresponds to a family of hyperarithmetical isomorphisms between computable algebras $\mathfrak{B} \uparrow\left(\bigcap_{i=1, \ldots, m} a_{i}^{\varepsilon_{i}}\right)$ and $\mathfrak{B} \uparrow\left(\bigcap_{i=1, \ldots, m} b_{i}^{\varepsilon_{i}}\right), \varepsilon_{1}, \ldots, \varepsilon_{m} \in\{0,1\}$, these Boolean algebras are not hyperarithmetically isomor-

phic for at least one sequence $\varepsilon_{1}, \ldots, \varepsilon_{m} \in\{0,1\}$. Then the elements $\bigcap_{i=1, \ldots, m} a_{i}^{\varepsilon_{i}}$ and $\bigcap_{i=1, \ldots, m} b_{i}^{\varepsilon_{i}}$ are automorphic but not hyperarithmetically automorphic in $\mathfrak{B}$. Note that in addition we have proven the existence of two isomorphic but not hyperarithmetically isomorphic Boolean algebras.

\section{References}

1. Barwise J., Admissible Sets and Structures, Springer-Verlag, Berlin (1975).

2. Ash C. J. and Knight J. F., Computable Structures and the Hyperarithmetical Hierarchy, Elsevier, Amsterdam (2000).

3. Ershov Yu. L. and Goncharov S. S., Constructive Models (Siberian School of Algebra and Logic), Kluwer Academic / Plenum Publishers, New York (2000).

4. Morozov A. S., "Functional trees and automorphisms of models," Algebra and Logic, 32, No. 1, 28-38 (1993).

5. Harrison J., "Recursive pseudo-well-ordering," Trans. Amer. Math. Soc., 131, $526-543$ (1968).

6. Barwise J. and Moschovakis Y. N., "Global inductive definability," J. Symbolic Logic, 43, No. 3, 521-534 (1978).

7. Sacks G. E., Higher Recursion Theory, Springer-Verlag, Heidelberg (1990).

8. Rogers L., "The structure of $p$-trees: algebraic systems related to Abelian groups," in: Abelian Group Theory. Proc. Second New Mexico State Univ. Conf. Las Cruces, N.M., 1976, Springer-Verlag, Berlin, 1977, pp. 57-72. (Lecture Notes in Math.; 616.)

9. Rogers H., Theory of Recursive Functions and Effective Computability, McGraw-Hill Book Company, New York; St. Louis; San Francisco; Toronto; London; Sydney (1967).

10. Fuchs L., Infinite Abelian Groups. Vol. 1, Academic Press, New York (1970).

11. Alaev P. E., "Scott ranks of Boolean algebras," in: Trudy Inst. Mat. (Novosibirsk), 1996, 30, pp. 3-25.

12. Goncharov S. S., "Constructivizability of superatomic Boolean algebras," Algebra and Logic, 12, No. 1, 17-22 (1973). 